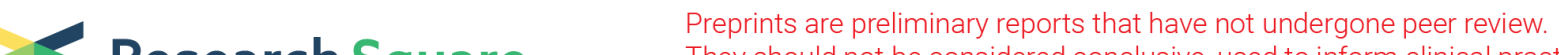 Research Square They should not be considered conclusive, used to inform clinical practice, or referenced by the media as validated information.
}

\section{Therapeutic effect of acetabular fractures using the Pararectus approach combined with 3D printing technique}

\section{Ruyi Zou}

The First Affiliated Hospital of Bengbu Medical College

Min Wu ( $\square$ wumin20020@163.com)

The First Affiliated Hospital of Bengbu Medical College https://orcid.org/0000-0003-0081-7611

Jianzhong Guan

The First Affiliated Hospital of Bengbu Medical College

\section{Yuzhou Xiao}

The First Affiliated Hospital of Bengbu Medical College

\section{Xiaotian Chen}

The First Affiliated Hospital of Bengbu Medical College

\section{Research Article}

Keywords: Pararectus approach, Acetabular fractures, 3D printing, Fracture fixation

Posted Date: March 5th, 2020

DOl: https://doi.org/10.21203/rs.2.23813/v2

License: (c) (1) This work is licensed under a Creative Commons Attribution 4.0 International License. Read Full License 


\section{Abstract}

Objective: To explore the clinical efficacy of pararectus approach combined with 3D printing technique for the surgical treatment of partial acetabular fractures.

Methods: We retrospectively evaluated 33 (20 males and 13 females) patients with acetabular fractures in the period of June 2017 to December 2018; According to judet-letenneal classification: 11 cases were of anterior column fracture, 10 cases were of double column fracture, 7 fractures were of the anterior column with posterior half transverse, 3 fractures were of transverse fracture, and 2 case was of "T" fracture. For all cases, 3D printing is used to print the acetabular model. Pre-bent reconstruction plates from the model were placed to fixate fractures via the pararectus approach.

Results : Thirty-three patients were followed up for 12-18 months (average,14 months); two patients (6.0\%) developed postoperative ossifying myositis, and there are no obvious symptoms at present; One patient (3.0\%) developed postoperative wound infection, and the wound completely improved by secretion culture, enhanced dressing and effective antibiotics; All the acetabular fractures united after 12 to 16 weeks(average,13 weeks);According to the modified Merle d'Aubigne and Postel scoring system to assess the hip function: excellent in 22 cases (66.7\%), good in 7 cases(21.2\%) and fair in 4 cases(12.1\%).

Conclusions: In the treatment of partial acetabular fractures, the pararectus approach combined with 3D printing technique can achieve effective reduction and fixation, decrease intraoperative hemorrhage, shorten operation time and the internal fixation position can be properly adjusted during the operation by looking directly at the model.

\section{Background}

Acetabular fractures are usually associated with some high-energy injuries. Due to its exceptional location, open reduction and internal fixation has become the "gold standard" for the treatment of displaced acetabular fractures.The position of the acetabulum is deep, and it is often difficult to expose fractures, especially complex acetabular fractures.

In the classic work of Judet and Letournel, the ilioinguinal approach was described in detail. Owing to this surgical approach can fully expose the front of the acetabulum and has always been a classic approach to the treatment of acetabular anterior fractures .However, this surgical method with a long incision and often needs to dissect important nerves, blood vessels and other structures, so the operation is complicated. Therefore, some scholars reported that the improved Stoppa was used as a small incision to replace the traditional ilioinguinal approach .A recent study showed that compared with the ilioinguinal approach, reduction and fixation of fractures can be achieved more easily by using the modified Stoppa approach.But, the Stoppa way still has certain limitations. For example, there is a specified distance from the iliac wing fracture. It is often to use a tiny incision on the iliac crest to assist in the reduction. At the 
same time, because of the small incision, it is difficult to operate in patients with severe displacement of the fracture, especially in obese patients.

To overcome the limitations of these conventional approaches, we used the pararectus approach described by Professor Keel .At the same time, in recent years, 3D printing technology has been increasingly utilized in cases of complex fractures. So, we use the pararectus approach in combination with 3D printing technology to treat complex acetabular fractures. According to the preoperative 3D printing model, the fracture morphology of the acetabulum can be visually observed and pre-bend of the steel plates before surgery.

\section{Materials And Methods}

This study was approved by the local Ethics Committee of our institution,and all patients gave informed consent and signed it. We found patients through the institution's case system and collected data through outpatient review. We proceeded to a retrospective analysis of 33 patients treated at our institution from 06/2017 to 12/2018.According to judet-letenneal classification: 11 cases were of anterior column fracture, 10 cases were of double column fracture, 7 fractures were of the anterior column with posterior half transverse, 3 fractures were of transverse fracture, and 2 case was of " $T$ " fracture. All patients were operated on by the Corresponding Author (Min Wu).Patients who fulfilled the following inclusion criteria: admissions were completed with pelvic film, pelvic CT scan $+3 \mathrm{D}$ reconstruction and 3D printing model; closed acetabular fracture; regular follow-up after discharge; Exclusion criteria included: open acetabular fracture; those with deformity and dysfunction of lower limbs before injury.All patients underwent a pelvic CT scan was performed to obtain the DICOM format data of the 3D reconstructed image of the pelvic thin layer, imported into Mimics10.01 software (Mate rialise company, Belgium) to generate a 3D virtual model of the pelvis; and then using fused deposition modeling (FDM) 1:1 scale printing to create a mirrored pelvic model(Fig. 1).Patient demographics are depicted in Table 1.

\section{Surgical technique}

Landmarks for the incision were the navel, pubic symphysis and the anterior superior iliac spine (ASIS), as professor Keel [13] descriptive. After general anesthesia, the incision start at the middle and outer $1 / 3$ of the line connecting the umbilical cord with the ASIS, and stop at the middle and inner $1 / 3$ of the line connecting the ASIS with pubic symphysis (Fig. 2). The length of this incision was about $9-14 \mathrm{~cm}$. Along the incision line, the skin, subcutaneous tissue and deep fascia were cut in turn. Palpate to identify the outer edge of rectus abdominous and cut the external oblique, internal oblique and transverse abdominal muscles along it.We can clearly see important neurovascular structures(Fig.3). Pay heed to avoid damaging the blood vessels and spermatic cord (or round ligament of the uterus). Through the retroperitoneal space, the peritoneum and the pelvic tissue are led to the inside. Other structures are led to the outside, and the real pelvic ring structure is exposed to the inside of the pelvis. Searching for the "death crown" above the obturator of the superior pubic branch and ligate it. 
According to the need for reduction of fractures, different windows are selected for exposure. The acetabular fractures reduction was completed with apical cone, reduction forceps and Kirschner wire during the operation. The Kirschner wire was used to temporarily fix the fracture block. According to the $3 \mathrm{D}$ printing model, the reconstruction plates and screw were selected at appropriate positions. When satisfied, leave the drainage tube and suture layer by layer.

\section{Evaluation}

All surgical data (blood loss, operative time, length of the incision, intraoperative and postoperative complications) were recorded. Patients were regularly followed up at one, three, six, and 12 months. All patients underwent anterior posterior (AP) and Judet oblique view x-rays projections of acetabular fractures, and pelvic CT+ three-dimensional reconstruction after surgery (Fig. 4), corresponding author (Wu Min) evaluated the x-rays of all patients, suggesting that fracture reduction is well. Clinical outcomes were evaluated using the modified Merle d'Aubigné score, ranging from excellent (18 points), good (1517 points), fair (14 or 13 points), and poor (<13 points).

\section{Results}

Thirty-three patients (mean age 48years; range, 35-63 years), included 20 men and 13 women, were treated successfully with open reduction and internal fixation by the pararectus approach. The average time of surgery was 203 min (range:135-245 min)and mean intraoperative blood loss was 1030ml(range:450-1400ml);33 patients were followed up for 12-18 months( average,14 months);The average length of the incision was $11 \mathrm{~cm}$ (range:9-14cm).two patients $(6.0 \%)$ developed postoperative ossifying myositis, and there are no obvious symptoms at present; One patient (3.0\%) developed postoperative wound infection, and the wound completely improved by secretion culture, enhanced dressing and effective antibiotics; all patients underwent pelvic film and pelvic CT+ three-dimensional reconstruction after surgery, suggesting that fractures reduction as well. All the acetabular fractures united after 12 to 16 weeks(average,13 weeks);According to the modified Merle d'Aubigne and Postel scoring system to assess the hip function:excellent in 22 cases (66.7\%), good in 7 cases(21.2\%) and fair in 4 cases(12.1\%).Specific data refer to Table 2

\section{Discussion}

The pararectus approach combined with 3D printing technique was introduced for treatment of acetabular fractures. The main suggested advantage in comparison to conventional approaches was to simplify the treatment of specific fracture patterns with less invasive tissue dissection. The pararectus approach can be used to fix the acetabular anterior and quadrilateral acetabular fractures under direct vision. Additionally, application of 3D printing technique can directly observe the fracture morphology of the acetabulum and pre-bend of the steel plates before surgery. 
Due to the special anatomical position of the acetabulum and its relationship with surrounding tissues, the anatomical reduction of the articular surface has become the treatment target for displaced acetabular fractures.Appropriate surgical approach can not only reduce the injury of the patient, shorten the operation time, but also realize the visualization of the fracture, which helps to the reduction and fixation of the fracture.

The pararectus approach, as reported by Keel in 2012, was used to treat acetabular fractures predominantly involving the anterior column and the quadrilateral plate .Compared with the traditional ilioinguinal approach and the modified Stoppa approach,the pararectus approach has the advantages of less trauma, no need to dissect important nerve and blood vessels, and less damage to tissues. At the same time, because the incision is located on the same side of the fracture, it is closer to the acetabulum and can look directly at the front of the acetabulum, which is of great significance for the reduction and fixation of the fracture.

Keel et al. reported that in the treatment of 48 cases of acetabular fractures, the pararectus approach was used to provide clear fracture visualization; the average incision length was $11 \mathrm{~cm}$, which reduced soft tissue damage;Bastian et al.Research shows that, compared with the modified Stoppa approach, the pararectus approach can reveal more false pelvis, and the posterior ring can be fixed without additional surgical approach.Mardian et al. reported that in the comparative study of the pararectus approach and the ilioinguinal approach, the pararectus approach was superior to the ilioinguinal approach in reducing the gap between the fracture blocks.

As the application of 3D printing technology in orthopedics becomes more and more mature, when we understand the classification and displacement of fracture, the process of getting rid of the traditional two-dimensional imaging data obtained only before surgery and constructing a three-dimensional morphology in mind.The 3D printed fracture model can be more convenient to observe the shape of the fracture block, which helps orthopedists to stereoscopically locate the acetabular fracture. At the same time, the application of 3D printing technology can enable orthopedic surgeons to pre-design the fracture reduction sequence, the position of the steel plate and the screwing angle of the screw according to the characteristics of the fracture. In the study by Tack $p$ and Martellini et al. the application of 3D printing technology reduced the patient's operation time, intraoperative blood loss, and also reduced intraoperative and postoperative complications .

When dealing with acetabular fractures, we often need a complete preoperative examination, preoperative evaluation, and choice of surgical approach. Attention should be paid to the treatment of acetabulum fracture by the pararectus approach: $\triangle \mathrm{A}$ single incision cannot be used for fractures of the posterior wall with acetabular joints, and a combined Kocher-Langenbeck approach is often required. For patients who need the Kocher-Langenbeck combined approach, adopt a "floating" position before disinfecting the towels, which is conducive to changing to the supine or lateral position as needed during the operation and reducing the operation time; $\mathbb{Z}$ For patients with severe extra peritoneal adhesions, consider using this surgical approach as appropriate; $₫$ The surgeon must be familiar with the anatomy of the abdomen, and 
in patients with peritoneal rupture, suture it in time;"Death crown" blood vessels are the anastomotic arteriovenous system of the inferior abdominal wall or the external iliac arteriovenous system and the obturator artery and vein. This surgical approach can view it directly above the medial obturator of the superior pubic branch, once found, it should be ligated to prevent the tear of the blood vessel caused by traction during fracture reduction, resulting in uncontrollable bleeding .

The main limitation of this study is its small size from a single institution. Owing to the relatively short follow-up time in this study, the mid-to-long-term clinical efficacy requires further follow-up of patients.

\section{Conclusions}

In summary, the pararectus approach provides sufficient space for acetabular fractures including the anterior column and the quadrilateral. Combined with 3D printing technique can achieve an effective reduction and fixation, decrease intraoperative hemorrhage, shorter operation time and the internal fixation position can be properly adjusted during the operation by looking directly at the model. Due to the short clinical practice of the pararectus approach, the number of reported cases is also quite limited, and a large number of postoperative follow-ups are lacking. The exact effect is yet to be verified.

\section{Abbreviations}

ASIS

Anterior superior iliac spine

\section{Declarations}

\section{Ethics approval and consent to participate}

This study was approved by the local Ethics Committee of our institution,and all patients gave informed consent and signed it.This study was done in agreement with the 1964 Helsinki declaration and its later amendments.

\section{Consent for publication}

All participants gave their written consent to participate in the study and for any possible publication.

\section{Availability of data and materials}

The materials described in the manuscript, including all relevant raw data,are available from the corresponding author upon request by email.

\section{Competing interests}

The authors declare that they have no competing interests. 


\section{Funding}

No funding was received.

\section{Authors' contributions}

$\mathrm{RYZ}, \mathrm{MW}$ and $\mathrm{XTC}$ collected the data, examined the patients,evaluated the radiology findings, and performed the statistical analysis. MW,JZG and YZX examined and operated on the patients, reviewed the manuscript,and planned the study.RYZ wrote the initial manuscript draft which was then revised appropriately by all authors.All authors read and approved the final submitted manuscript.

\section{Acknowledgments}

Not applicable

\section{References}

1. Triantaphillopoulos P G, Panagiotopoulos E C, Mousafiris C, et al. Long-term results in surgically treated acetabular fractures through the posterior approaches.[J]. Journal of Trauma, 2007, 62(2):378.

2. Harris A M, Althausen P, Kellam J F, et al. Simultaneous anterior and posterior approaches for complex acetabular fractures[J]. Journal of Orthopaedic Trauma, 2008, 22(7):494-7.

3. Tibbs $B$ M , Kopar $P$, Dente $C \mathrm{~J}$, et al. Acetabular and isolated pelvic ring fractures: a comparison of initial assessment and outcome[J]. American Surgeon, 2008, 74(6):538-41; discussion 541.

4. Letournel $\mathrm{E}$. The treatment of acetabular fractures through the ilioinguinal approach[J]. Clin Orthop Relat Res, 1993, 292(292):62-76.

5. Schmidtrohlfing B, Reilmann H, Pape H C. [Fractures of the acetabulum. Diagnostic and therapeutic strategies].[J]. Der Unfallchirurg, 2010, 113(3):217-229.

6. Gänsslen A, Grechenig S T, Nerlich M, et al. Standard Approaches to the Acetabulum Part 2: Ilioinguinal Approach[J]. Acta Chirurgiae Orthopaedicae Et Traumatologiae Cechoslovaca, 2016, 83(4):217-222.

7. Keel M J B, Tomagra S, Bonel H M, et al. Clinical results of acetabular fracture management with the Pararectus approach[J]. Injury-international Journal of the Care of the Injured, 2014, 45(12):19001907.

8. Andersen R C, O'Toole R V, Nascone J W, et al. Modified stoppa approach for acetabular fractures with anterior and posterior column displacement: quantification of radiographic reduction and analysis of interobserver variability.[J]. Journal of Orthopaedic Trauma, 2010, 24(5):271-8.

9. Bastian J D, Tannast M, Siebenrock K A, et al. Mid-term results in relation to age and analysis of predictive factors after fixation of acetabular fractures using the modified Stoppa approach[J]. Injuryinternational Journal of the Care of the Injured, 2013, 44(12):1793-1798. 
10. Cole J D, Bolhofner B R. Acetabular fracture fixation via a modified Stoppa limited intrapelvic approach. Description of operative technique and preliminary treatment results.[J]. Clinical Orthopaedics \& Related Research, 1994, (305)(305):112.

11. Hirvensalo E, Lindahl J, Böstman O. A new approach to the internal fixation of unstable pelvic fractures.[J]. Clin Orthop, 1993, 297(297):28-32.

12. Shazar N, Eshed I, Ackshota N, et al. Comparison of acetabular fracture reduction quality by the ilioinguinal or the anterior intrapelvic (modified Rives-Stoppa) surgical approaches.[J]. Journal of Orthopaedic Trauma, 2014, 28(6):313.

13. Keel M J, Ecker T M, Cullmann J L, et al. The Pararectus approach for anterior intrapelvic management of acetabular fractures: an anatomical study and clinical evaluation[J]. Journal of Bone \& Joint Surgery British Volume, 2012, 94(3):405.

14. Briffa N, Pearce R, Hill A M, et al. Outcomes of acetabular fracture fixation with ten years' followup[J]. Journal of Bone \& Joint Surgery-british Volume, 2011, 93(2):229-236.

15. Ferguson T A, Patel R, Bhandari M, et al. Fractures of the acetabulum in patients aged 60 years and older: an epidemiological and radiological study.[J]. Journal of Bone \& Joint Surgery American Volume, 2010, 92(2):250-257.

16. Tannast M, Najibi S, Matta J M. Two to twenty-year survivorship of the hip in 810 patients with operatively treated acetabular fractures.[J]. Journal of Bone \& Joint Surgery American Volume, 2012, 94(17):1559.

17. Mardian, S, Schaser, K. D, Hinz, P, et al.. Fixation of acetabular fractures via the ilioinguinal versus pararectus approach: a direct comparison[J]. Bone \& Joint Journal,2015, 97-B(9):1271-1278.

18. Bastian J D, Savic M, Cullmann J L, et al. Surgical exposures and options for instrumentation in acetabular fracture fixation: Pararectus approach versus the modified Stoppa.[J]. Injury-international Journal of the Care of the Injured, 2016, 47(3):695-701.

19. Tack P, Victor J, Gemmel P, et al. 3D-printing techniques in a medical setting: a systematic literature review[J]. Biomedical Engineering Online, 2016, 15(1):115.

20. Martelli N, Serrano C, Van d B H, et al. Advantages and disadvantages of 3-dimensional printing in surgery: A systematic review.[J]. Surgery, 2016, 159(6):1485-1500.

21. Ponsen $K$ J, Joosse $P$, Schigt $A$, et al. Internal fracture fixation using the Stoppa approach in pelvic ring and acetabular fractures: technical aspects and operative results[J]. Journal of Trauma \& Acute Care Surgery, 2006, 61(3):662-667.

22. Kandhari V K, Desai M M , Bava S S, et al. Avascular Necrosis of Acetabulum: The Hidden Culprit of Resistant Deep Wound Infection and Failed Fixation of Fracture Acetabulum - A Case Report[J]. Journal of Orthopaedic Case Reports, 2015, 5(4):36-39.

23. Al W T. A new concept and classification of corona mortis and its clinical significance:[J]. Chinese Journal of Traumatology, 2016, 19(5):S100812751630195X.

24. Darmanis S, Lewis A, Mansoor A, et al. Corona mortis: An anatomical study with clinical implications in approaches to the pelvis and acetabulum[J]. Clinical Anatomy, 2010, 20(4):433-439. 


\section{Tables}

Table 1 Patient demographics

\begin{tabular}{lll}
\hline Parameter & Value & Percent(\%) \\
\hline Mean age(years) & $48(35-63)$ & \\
Gender & & \\
$\quad$ Male & 20 & 60.6 \\
$\quad$ Female & 13 & 39.4 \\
Mechanism of injury & & \\
$\quad$ Road traffic accident & 18 & 54.6 \\
Fall & 11 & 33.3 \\
Heavy object injury & 4 & 12.1 \\
Fracture classification & & \\
Anterior column & 11 & 33.3 \\
Both columns & 10 & 30.3 \\
Anterior column and posterior hemitransverse & 7 & 21.2 \\
Transverse & 3 & 9.1 \\
T-Type & 2 & 6.1 \\
Mean delay to surgery (days) & $9(6-17)$ & \\
\hline
\end{tabular}

Table 2 Postoperative outcomes 


\begin{tabular}{lll}
\hline Postoperative data & Value & Percent \\
\hline Mean blood loss(ml) & 1030 (range:450-1400) & \\
Mean operative time(min) & 203(range:135-245) & \\
Mean length of incision(cm) & 11 (range:9-14) & \\
Mean union time(weeks) & 13 (range:12-16) & \\
Postoperative complications & & 6.1 \\
postoperative ossifying myositis & 2 & 3.0 \\
postoperative wound infection & 1 & 66.7 \\
The modified Merle d'Aubigné score & & 21.2 \\
Excellent & 22 & 12.1 \\
Good & 7 & \\
Fair & 4 & \\
\end{tabular}

Figures 

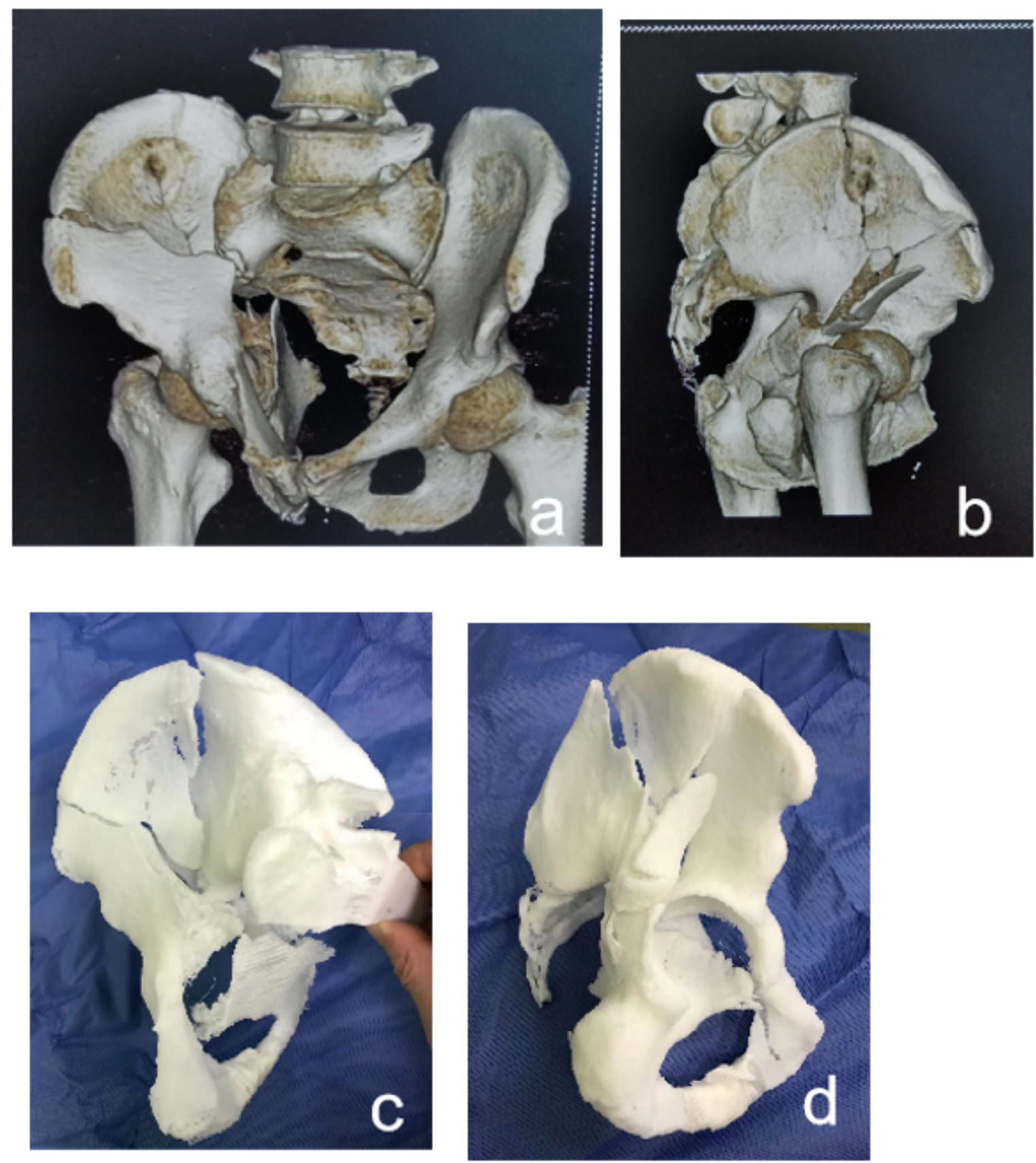

Figure 1

(a,b) A 45-year-old male patient suffered a road-traffic accident. The fracture was diagnosed as a double column fracture through took CT scan $+3 D$ reconstruction. (c,d) 3D printed model made from data obtained as a result of CT scan. 


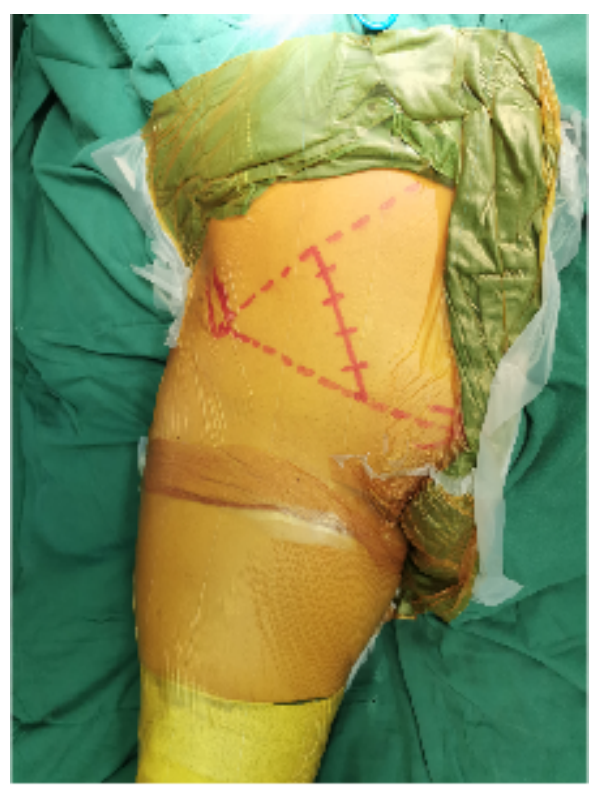

Figure 2

Landmarks and the skin incision of the pararectus approach

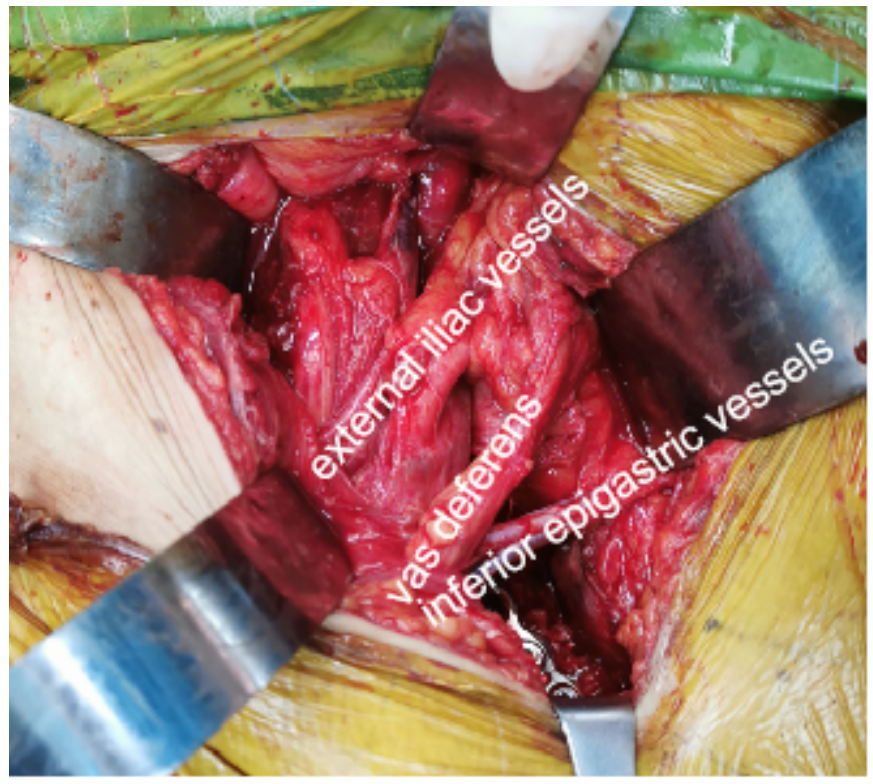

\section{Figure 3}

Intra-operative photograph (right hemipelvis) showed by the pararectus approach with the external iliac vessels, the vas deferens and the inferior epigastric vessels.

政

\section{Figure 4}

Postoperative anteroposterior (AP) (see Fig 4a) and Judet oblique view x-rays (see Figs $4 \mathrm{~b}$ to $4 \mathrm{c}$ ), and computed tomography (CT) scan (see Fig 4d) of the acetabulum shows the outcome of reduction of the fracture. 